\title{
Clinical Outcome of Septic Patients With Heart Failure With Preserved Ejection Fraction Presenting to the Emergency Department of a Tertiary Hospital: A Retrospective Cohort Study
}

Ralphe Bou Chebl, Iskandar Berbari, Rawan Safa, Ali Shami, Mohammad Sabra, Sarah Jamali, Maha Makki, Hani Tamim and Gilbert Abou Dagher*

Department of Emergency Medicine, American University of Beirut Medical Center, Beiru, Lebanon

Background: Patients with heart failure with preserved ejection fraction (HFpEF) may be at a higher risk of mortality from sepsis than patients without heart failure.

OPEN ACCESS

Edited by:

Mitja Lainscak

University of Ljubljana, Slovenia

Reviewed by:

Yongan Xu

Zhejiang University, China

E. Wang,

Central South University, China

*Correspondence:

Gillbert Abou Dagher ga66@aub.edu.lb

Specialty section: This article was submitted to Intensive Care Medicine and

Anesthesiology,

a section of the journal

Frontiers in Medicine

Received: 06 December 2019 Accepted: 06 October 2020 Published: 30 October 2020

Citation:

Chebl RB, Berbari I, Safa R, Shami A, Sabra M, Jamali S, Makki M, Tamim H and Dagher GA (2020) Clinical

Outcome of Septic Patients With Heart Failure With Preserved Ejection Fraction Presenting to the Emergency Department of a Tertiary Hospital: A Retrospective Cohort Study. Front. Med. 7:517999.

doi: 10.3389/fmed.2020.517999
Objective: The aim of this study is to compare sepsis-related morbidity and mortality between patients with HFpEF and patients without heart failure presenting to the emergency department (ED) of a tertiary medical center.

Design: Single-center retrospective cohort study conducted at an academic ED between January 1, 2015 and December 31, 2018.

Patients: Patients with a diagnosis of sepsis were included.

Main Measures: Bivariate and multivariate analyses were performed to look at differences in demographics, infection, and treatment parameters as well as outcomes of patients with sepsis. The primary outcome of the study was in-hospital mortality. Secondary outcomes included ED mortality, lengths of stay, and treatment differences between both groups.

Key Results: A total of 1,092 patients presented with sepsis to the ED, of which 305 (27.93\%) had HFpEF. There was no significant difference in in-hospital mortality between the two groups ( $40.7 \%$ vs. $37.4 \% ; p=0.314$ ). However, there was a significant increase in ED mortality for septic HFpEF patients compared to non-heart failure patients (2.4 vs. $0.4 \% ; p=0.003)$. Septic HFpEF patients presenting to the ED were older than non-heart failure patients (76.84 vs. 68.44 years old; $p<0.0001$ ). On the other hand, there was no significant increase in the use of vasopressors in the first $24 \mathrm{~h}$ between both groups. There was a significantly higher rate of intubation in the first $48 \mathrm{~h}$ for septic HFpEF patients (17.5 vs. $8.9 \% ; p<0.0001)$. Finally, there was significantly less intravenous fluid requirement at $6 \mathrm{~h}(1.94 \mathrm{~L}$ vs. $2.41 \mathrm{~L} ; p<0.0001)$ and $24 \mathrm{~h}(3.11 \mathrm{~L}$ vs. 3.54L; $p=0.004)$ for septic patients with HFpEF compared to non-heart failure patients.

Conclusion: Septic HFpEF patients experienced an increase in ED mortality, intubation, and steroid use compared to septic non-heart failure patients.

Keywords: heart failure, diastolic, sepsis, mortality, emergency department 


\section{INTRODUCTION}

Heart failure and sepsis are major public health problems. More than 5.8 million in the United States and 23 million people worldwide have heart failure (1). The prevalence of heart failure with preserved ejection fraction (HFpEF) relative to heart failure with reduced ejection fraction (HFrEF) is rising at an alarming rate of $1 \%$ per year, with $50 \%$ of heart failure patients currently having $\operatorname{HFpEF}(2,3)$. Morbidity, mortality, and healthcare costs of HFpEF are on par with HFrEF (4). Sepsis, on the other hand, affects more than 600,000 patients in the USA each year and is associated with high mortality: up to $70 \%$ in seriously ill patients $(5-7)$. It is a condition that is caused by the host's response to a bacterial infection. The cytokines released affect multiple organs, including the cardiovascular system (6). Sepsis has been shown to cause myocardial suppression, as well as diastolic dysfunction and decreased cardiac index (8). HFpEF patients may be at a higher risk of mortality from sepsis than patients without heart failure due to insufficient cardiovascular reserves during systemic infection (8). There is limited data available on the association between heart failure with preserved Ejection Fraction with the clinical outcome and mortality rates in sepsis and septic shock (9). The aim of this study is to compare sepsis-related morbidity and mortality between patients with HFpEF and patients without heart failure presenting to a tertiary medical center.

\section{METHODS}

\section{Study Design and Setting}

This was a single-center, retrospective, cohort study conducted in an academic emergency department (ED) between January 1, 2015, and December 31, 2018. The hospital's electronic system was used to query patients' charts. All demographic information, including comorbidities, vital signs, laboratory results, and resuscitation parameters, were extracted from scanned charts by research fellows. Multiple meetings with the principal investigators were conducted to standardize the data extraction and entry process. This study was approved by the

\footnotetext{
Abbreviations: $\mathrm{cu} . \mathrm{mm}$, per cubic millimeter; \%, percentage; $\mathrm{AF}$, atrial fibrillation; ASE, American Society of Echocardiography; Bpm, beats per minute; BUN, blood urea nitrogen; cc/kg, cubic centimeters per kilogram; CI, Confidence interval; CKD, chronic kidney disease; COPD, chronic obstructive pulmonary disease; DBP, diastolic blood pressure; EACVI, European Association of Cardiovascular Imaging; E. coli, Escherichia coli; ED, emergency department; EHR, electronic health records; ESRD, end stage renal disease; g/dL, gram per deciliter; g/L, grams per liter; GPU, general practitioner unit; H, hours; HFpEF, heart failure with preserved ejection fraction; HFrEF, heart failure with reduced ejection fraction; HR, heart rate; ICD, International Statistical Classification of Diseases; ICU, intensive care unit; INR, International Normalized Ratio; IQR, interquartile range; IRB, institutional review board; IV, intravenous; L, liter; LOS, length of stay; LV, left ventricular; $\mathrm{mg} / \mathrm{dL}$, milligrams per deciliter; $\mathrm{mmHg}$, millimeter of mercury; $\mathrm{mmol} / \mathrm{L}$, millimoles per liter; $\mathrm{ng} / \mathrm{ml}$, nanogram per milliliter; NT-proBNP, NTpro B-type natriuretic peptide; $\mathrm{OR}$, odd ratio; $\mathrm{PaCO}_{2}$, partial pressure of carbon dioxide; PCWP, pulmonary capillary wedge pressure; $\mathrm{pg} / \mathrm{ml}$, picogram $/ \mathrm{milliliter}$; PT, prothrombin time; PTT, partial thromboplastin time; SBP, systolic blood pressure; SD, Standard deviation; Sec, second; SOFA, Sequential [Sepsis-related] Organ Failure Assessment; SPSS, Statistical Package for Social Sciences; SV, stroke volume; TIA, transient ischemic attack; USA, United states of America; $\chi 2$, Chi square test.
}

Institutional Review Board of the American University of Beirut and the hospital's IRB (BIO-2018-0106) and carried out in accordance with the recommendations provided. The research was performed according to ethical principles and in compliance with all prevailing and applicable laws, rules, and regulations and policies regarding the protection of human subjects and research conduct as outlined by the declaration of Helsinki. Subject privacy and data confidentiality were of paramount concern at all times, and every effort was made to protect subjects' rights and welfare.

\section{Patient Selection}

Patients' ED encounters were filtered by an experienced data user using the hospital's EHR (electronic health records) via an extensive structured keyword search and ICD-9 coding (International Statistical Classification of Diseases, Ninth Revision and Related Health Problems). The ICD-9 diagnoses retrieved were sepsis (995.91) and septic shock (785.52). A list of all patients who were diagnosed with sepsis or septic shock between January 1, 2015, and December 31, 2018, was therefore compiled (IRB \#BIO-2018-0106). Sepsis was defined according to the sepsis-3 definition as the presence of an infection with signs of organ dysfunction, which were represented by an increase in the Sequential [Sepsis-related] Organ Failure Assessment (SOFA) score of two points or more. Septic shock was defined by a vasopressor requirement to maintain a mean arterial pressure of $65 \mathrm{~mm} \mathrm{Hg}$ and a serum lactate level $>2 \mathrm{mmol} / \mathrm{L}(>18 \mathrm{mg} / \mathrm{dL})$ in the absence of hypovolemia (10). Patients younger than 18 years, cardiac arrest patients, patients transferred from another hospital, trauma patients, pregnant patients, and HFrEF patients as well as patients who did not have an echocardiogram were excluded from the study. We elected to exclude patients with a reduced ejection fraction as these patients usually have high left ventricular (LV) filling pressures and often have diastolic dysfunction, and our aim was to compare patients with $\mathrm{HFpEF}$ to patients with a normal cardiac function and echo.

\section{Exposure: Heart Failure With Preserved Ejection Fraction}

Patients who were included in the study were stratified according to the presence of HFpEF. HFpEF was identified via the revision of echocardiography reports performed by cardiologists at our institution. The echocardiography report was considered valid if the procedure was performed at most 1 month before the admission date. Heart failure with preserved ejection fraction was defined according to the recommendation given by the American Society of Echocardiography (ASE) and the European Association of Cardiovascular Imaging (EACVI) (11). In order for patients to meet the definition, they had to meet three criteria. First, signs and/or symptoms of heart failure had to be present (11). Second, normal systolic LV function (LV ejection fraction $>50 \%$ ) should be present on echocardiography (11). Third, evidence of LV diastolic dysfunction had to also be present. According to the ASE/EACVI guidelines, diastolic dysfunction was based on four variables: Septal $\mathrm{e}^{\prime}<7 \mathrm{~cm} / \mathrm{s}$ or Lateral $\mathrm{e}^{\prime}<10 \mathrm{~cm} / \mathrm{s}$, an average E/ $/ \mathrm{e}^{\prime}>14$, left atrium (LA) volume index $>34 \mathrm{~mL} / \mathrm{m}^{2}$ and peak tricuspid regurgitation (TR) velocity 
$>2.8 \mathrm{~m} / \mathrm{s}$ (11). All these variables are routinely reported in echocardiograms at our institution.

\section{Outcome Measures}

The aim was to characterize the presentation of sepsis among HFpEF patients compared to those without heart failure and assess associated clinical outcomes. The primary outcome of the study was in-hospital mortality. Secondary outcomes included ED mortality, lengths of stay, and differences in treatment interventions between both groups. The infection site was determined from documentation in the medical record, culture results (blood, sputum, urine, other fluids), and/or radiology reports (such as chest X-rays). Laboratory results were obtained from the hospital's EHR system. Information about antibiotics and vasopressors, time to their initiation, and duration of their use was obtained by reviewing the scanned ED order sheet. Disposition status was also recorded by reviewing admission and discharge documents.

\section{Statistical Analysis}

Statistical Package for Social Sciences (SPSS) version 25.0 (Armonk, New York, USA: IBM Corp) was used to perform all statistical analyses. Continuous and categorical variables were presented as mean \pm SD and frequency/percentages, respectively. The Shapiro-Wilk test, Kurtosis and Skewness Z-score, and visualization of histograms were used to check for normality of distribution of all continuous variables. Time to antibiotics was presented as median and interquartile range (IQR). The different parameters were then stratified by whether or not patients had HFpEF (HFpEF only or no HFpEF and no HFrEF). Pearson's $\chi 2$ test was used to assess for statistical significance for the categorical variables, while the Student's $t$-test and the MannWhitney $U$-test were used for the continuous ones. Tests were interpreted at a significance level alpha $=0.05$. Multivariable analyses were performed to ascertain the association between HFpEF status and mortality [one for in-hospital mortality (Table 5) and another for ED mortality (Table 6)] in the septic population via a logistic regression. Another multivariate analysis was performed to determine the association between total LOS of in-hospital survivors and HFpEF via a linear regression (Table 7). A backward selection procedure, with the significance level for variable removal from the model set at 0.05 , was conducted. All clinically and statistically significant variables in the bivariate

TABLE 1 | Patients characteristics at presentation.

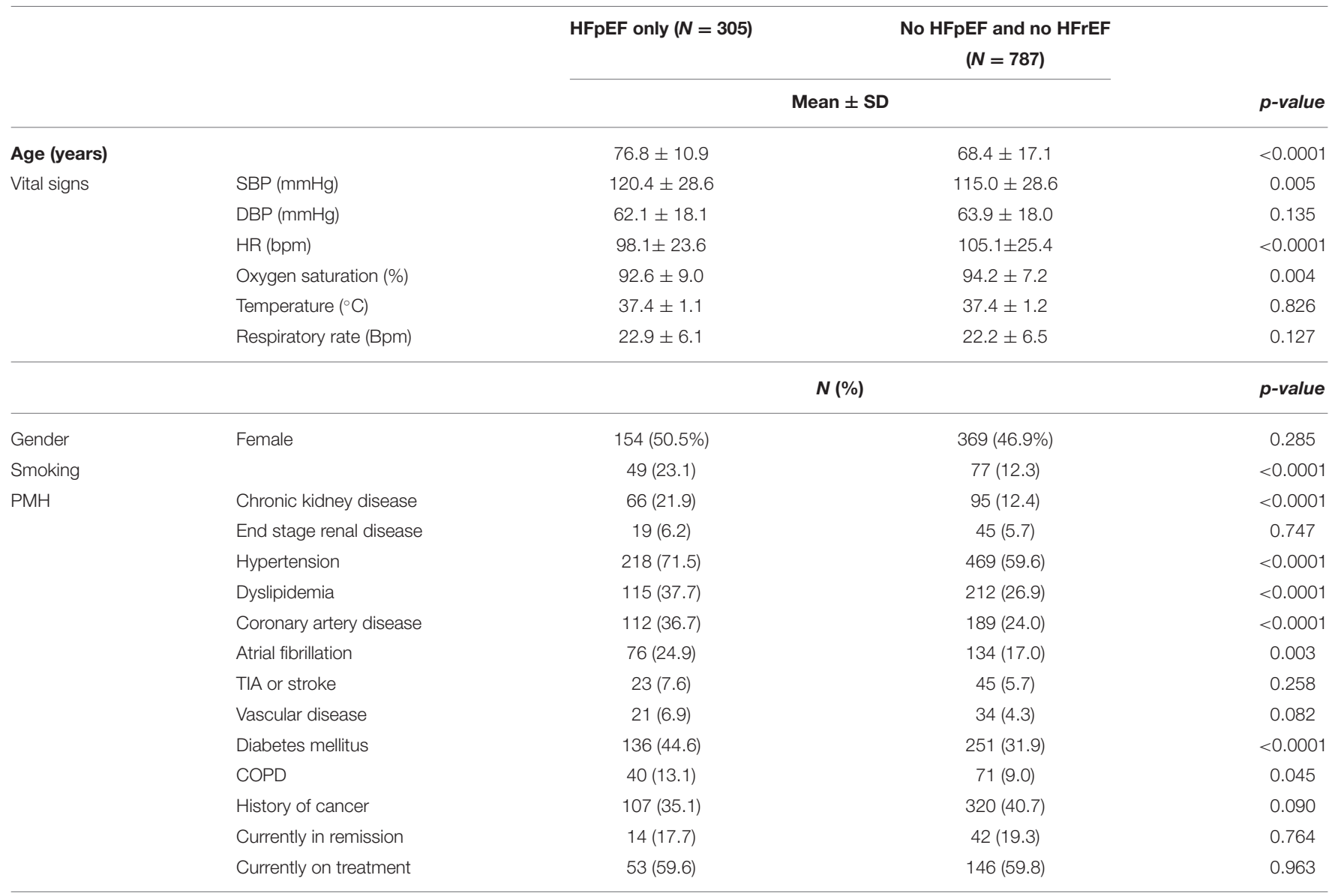

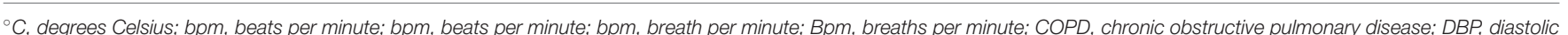

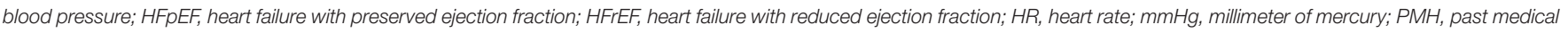
history; SBP, systolic blood pressure; SD, standard deviation. 
analysis level in were included in the multivariate analysis. The variables included in the model are listed in each table. The results were described as odd ratios (ORs) and their corresponding 95\% CI.

\section{RESULTS}

\section{Patient Characteristics at Presentation to the ED}

One thousand ninety-two septic patients were included in this study, of which 305 (27.93\%) had HFpEF on admission. On the other hand, 787 patients $(72.07 \%)$ had neither HFpEF nor HFrEF. There was an equal gender distribution among patients with HFpEF (50.5 vs. $49.5 \%$; $p=0.285$ ). Patients with HFpEF were significantly older than non-heart failure patients (76.84 vs. 68.44 years old; $p<0.0001$ ). The HFpEF group had a significantly higher rate of chronic kidney disease (21.9 vs. $12.4 \%$; $p<$ 0.0001 ), hypertension ( 71.5 vs. $59.6 \%$; $p<0.0001$ ), dyslipidemia
(37.7 vs. $26.9 \% ; p<0.0001$ ), coronary artery disease (36.7 vs. $24.0 \% ; p<0.0001)$, atrial fibrillation (24.9 vs. $17.0 \% ; p=0.003)$, diabetes mellitus (44.6 vs. $31.9 \% ; p<0.0001$ ), and chronic obstructive pulmonary disease (COPD) (13.1 vs. $9.0 \%$; $p=$ 0.045). Table 1 summarizes the patient's comorbidities. The most common symptom for HFpEF patients was dyspnea on exertion, which was seen in $80 \%$ of patients, followed by orthopnea and paroxysmal nocturnal dyspnea which were seen in 65 and $35 \%$ of patients. As for the signs, $85 \%$ of patients were found to have pitting edema, $75 \%$ were found to have crackles on auscultation.

\section{Vital Signs and Laboratory Tests}

On presentation to the ED, HFpEF patients had a significantly higher systolic blood pressure (120.47 vs. $114.99 \mathrm{mmHg} ; p=$ 0.005 ), a significantly lower heart rate (98.26 vs. $105.11 \mathrm{bpm} ; p$ $<0.0001$ ), and a significantly lower oxygen saturation (92.57 vs. $94.23 \% ; p=0.004)$. The rest of the vital signs at presentation were similar between the two cohorts (Table 1).

TABLE 2 | Laboratory results at presentation.

\begin{tabular}{|c|c|c|c|}
\hline & $\begin{array}{l}\text { HFpEF only } \\
\qquad(N=305)\end{array}$ & $\begin{array}{l}\text { No HFpEF and no HFrEF } \\
\qquad(N=787)\end{array}$ & \multirow[b]{2}{*}{ p-value } \\
\hline & \multicolumn{2}{|c|}{ Mean \pm SD } & \\
\hline Lactate at presentation $\mathrm{mmol} / \mathrm{L}$ & $3.5 \pm 2.7$ & $3.7 \pm 3.0$ & 0.444 \\
\hline Lactate second variable $\mathrm{mmol} / \mathrm{L}$ & $3.1 \pm 3.9$ & $3.2 \pm 3.4$ & 0.781 \\
\hline Albumin at presentation $\mathrm{g} / \mathrm{L}$ & $28.0 \pm 6.5$ & $27.6 \pm 6.8$ & 0.411 \\
\hline Procalcitonin ng/ml & $9.6 \pm 20.1$ & $14.2 \pm 29.6$ & 0.079 \\
\hline Glucose mg/dL & $156.4 \pm 76.3$ & $151.8 \pm 87.0$ & 0.471 \\
\hline Hemoglobin g/dL & $10.9 \pm 1.9$ & $11.0 \pm 2.3$ & 0.498 \\
\hline Hematocrit \% & $32.9 \pm 6.2$ & $33.0 \pm 7.0$ & 0.883 \\
\hline BUN mg/dl & $38.5 \pm 30.6$ & $35.5 \pm 27.7$ & 0.119 \\
\hline Creatinine mg/dl & $1.8 \pm 1.5$ & $1.7 \pm 1.8$ & 0.667 \\
\hline Baseline creatinine (for patients with CKD) mg/dL & $1.9 \pm 0.6$ & $1.8 \pm 0.6$ & 0.623 \\
\hline Sodium mmol/L & $135.6 \pm 6.5$ & $135.3 \pm 6.5$ & 0.496 \\
\hline Absolute neutrophil count /cu.mm & $10,439.6 \pm 7,650.1$ & $10,615.4 \pm 8,627.8$ & 0.756 \\
\hline Lymphocyte count \% & $13.9 \pm 17.3$ & $12.4 \pm 14.9$ & 0.194 \\
\hline WBC/cu.mm & $13,030.9 \pm 9,163.8$ & $13,362.4 \pm 10,946.7$ & 0.640 \\
\hline Bicarbonate mmol/L & $21.9 \pm 7.2$ & $21.2 \pm 5.6$ & 0.105 \\
\hline Chloride $\mathrm{mmol} / \mathrm{L}$ & $97.1 \pm 7.9$ & $97.2 \pm 7.1$ & 0.763 \\
\hline Bilirubin total mg/dL & $1.3 \pm 2.9$ & $1.6 \pm 3.1$ & 0.319 \\
\hline Troponin ng/mL & $0.1 \pm 0.1$ & $0.1 \pm 0.2$ & 0.676 \\
\hline Potassium mmol/L & $4.6 \pm 2.7$ & $4.7 \pm 6.2$ & 0.712 \\
\hline Magnesium mg/dL & $1.9 \pm 0.4$ & $2.2 \pm 6.3$ & 0.311 \\
\hline Calcium mg/dL & $8.6 \pm 0.9$ & $8.6 \pm 1.1$ & 0.932 \\
\hline Phosphate mg/dL & $3.4 \pm 1.5$ & $3.7 \pm 1.8$ & 0.022 \\
\hline Ph (arterial) & $7.36 \pm 0.12$ & $7.34 \pm 0.11$ & 0.098 \\
\hline $\mathrm{PaCO}_{2} \mathrm{mmHg}$ & $38.3 \pm 20.2$ & $35.9 \pm 14.2$ & 0.175 \\
\hline PT sec & $18.69 \pm 11.77$ & $20.49 \pm 16.25$ & 0.185 \\
\hline PTT sec & $33.88 \pm 16.58$ & $34.66 \pm 19.48$ & 0.653 \\
\hline INR & $1.6 \pm 1.0$ & $1.9 \pm 2.6$ & 0.259 \\
\hline
\end{tabular}

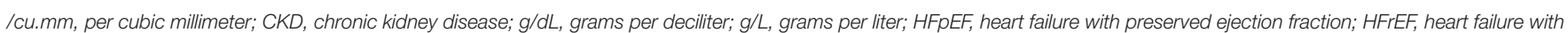

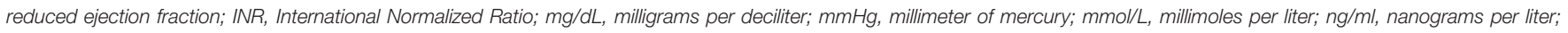
$\mathrm{PaCO}_{2}$, partial pressure of carbon dioxide; PT, prothrombin time; PTT, partial thromboplastin time; SD, standard deviation; sec, seconds; WBC, white blood cells. 
As for the laboratory studies at presentation, the two groups had similar lactate, albumin, hemoglobin, hematocrit, BUN, creatinine, sodium, absolute neutrophil count, lymphocyte count, white blood cell count, chloride, total bilirubin, troponin, potassium, magnesium, calcium, procalcitonin, $\mathrm{pH}$, bicarbonate, $\mathrm{PaCO}_{2}, \mathrm{PT}, \mathrm{PTT}$, and INR. However, patients with HFpEF had a significantly lower phosphate level (3.44 vs. $3.69 \mathrm{mg} / \mathrm{dL} ; p$ $=0.022$ ) (Table 2). The median value for pro-BNP for HFpEF patients was 955 with an IQR of 1,585.

\section{Patient Hospital Course}

HFpEF patients had a slight increase in in-hospital mortality ( 40.7 vs. $37.4 \%$; $p=0.314$ ); however, this difference was not statistically significant. Moreover, there was a significantly higher rate of ED mortality in the HFpEF cohort (2.4 vs. $0.4 \%$; $p=$ 0.003). During their hospital course, patients with HFpEF had a lower amount of fluid administered during the first $6 \mathrm{~h}(1.94 \mathrm{~L}$ vs. $2.41 \mathrm{~L} ; p<0.0001)$ and $24 \mathrm{~h}$ since $\mathrm{ED}$ admission $(3.11 \mathrm{~L}$ vs. $3.54 \mathrm{~L} ; p=0.004)$ compared to patients without HFpEF. Among survivors, the hospital length of stay was significantly lower for patients with $\mathrm{HFpEF}$ compared to non-heart failure patients (257.78h vs. $317.36 \mathrm{~h} ; p=0.03$ ). Over the first $24 \mathrm{~h}$, more HFpEF patients received steroids ( 23.4 vs. $18.1 \%$; $p=0.048$ ). Moreover, over the first $48 \mathrm{~h}$, a higher number of HFpEF patients were intubated compared to patients without heart failure (17.5 vs. 8.9\%; $p<0.0001$ ) (Table 3).

\section{Infection Characteristics}

There was no difference in the percentage of patients receiving antibiotics in the two groups. Time to antibiotic administration was also similar between the two cohorts (median: $2.1 \mathrm{~h}$ vs. $2.25 \mathrm{~h}$; interquartile range and interquartile range (IQR) $2.85 \mathrm{~h}$ vs. $3.86 \mathrm{~h}$ ). In both groups, the most common site of infection was the lungs. However, urinary tract infections were more common in HFpEF septic patients compared to septic patients without HFpEF (40.2 vs. $29.2 \%$; $p=0.001$ ). Moreover, HFpEF patients had less gastrointestinal infections when compared to the nonheart failure group (10.6 vs. $17.8 \%$; $p=0.004$ ). Bacteria were found mostly in blood in the two groups. However, there were more bacteria isolated from the urine in the HFpEF cohort (33.9 vs. $27.3 \% ; p=0.032$ ). The most common bacteria found in blood, urine, and wound, in both groups, was E. coli. The most common bacteria found in sputum was Pseudomonas aeruginosa and Acinetobacter baumannii in the HFpEF group and E. coli in the non-heart failure group (Table 4).

\section{Multivariate analysis}

Multivariate analyses were conducted to determine the association between HFpEF and hospital mortality, HFpEF

TABLE 3 | Patient hospital course.

\begin{tabular}{|c|c|c|c|c|c|}
\hline & & & $\begin{array}{l}\text { HFpEF only } \\
(N=305)\end{array}$ & $\begin{array}{l}\text { No HFpEF and no HFrEF } \\
\qquad(N=787)\end{array}$ & \multirow{3}{*}{$\begin{array}{c}\text { p-value } \\
<0.0001\end{array}$} \\
\hline & & & \multicolumn{2}{|c|}{ Mean \pm SD } & \\
\hline \multirow[t]{2}{*}{ IV fluids (L) } & \multicolumn{2}{|c|}{ IV fluid given during the first $6 \mathrm{~h}$} & $1.94 \pm 1.74$ & $2.41 \pm 1.82$ & \\
\hline & \multicolumn{2}{|c|}{ IV fluid given in the first $24 \mathrm{~h}$} & $3.11 \pm 2.23$ & $3.54 \pm 2.19$ & 0.004 \\
\hline \multirow[t]{7}{*}{ LOS } & \multirow[t]{3}{*}{ LOS in the ED (h) } & Overall & $15.77 \pm 18.86$ & $16.66 \pm 21.93$ & 0.533 \\
\hline & & Survivors & $15.46 \pm 18.71$ & $16.66 \pm 21.94$ & 0.40 \\
\hline & & Non-survivors & $29.16 \pm 21.71$ & $17.61 \pm 22.68$ & 0.47 \\
\hline & \multirow[t]{3}{*}{ Total hospital LOS (h) } & Overall & $349.98 \pm 391.15$ & $392.64 \pm 581.29$ & 0.238 \\
\hline & & Survivors & $257.78 \pm 256.94$ & $317.36 \pm 457.21$ & 0.03 \\
\hline & & Non-survivors & $484.58 \pm 500.76$ & $518.87 \pm 727.95$ & 0.63 \\
\hline & & & & & p-value \\
\hline \multirow[t]{5}{*}{ Medication used } & \multicolumn{2}{|c|}{ Norepinephrine given in the first $24 \mathrm{~h}$} & $95(31.1)$ & 207 (26.3) & 0.108 \\
\hline & \multicolumn{2}{|c|}{ Dopamine given in the first $24 \mathrm{~h}$} & $7(2.3)$ & $9(1.1)$ & 0.155 \\
\hline & \multicolumn{2}{|c|}{ Epinephrine given in the first $24 \mathrm{~h}$} & $6(2.0)$ & $22(2.8)$ & 0.437 \\
\hline & \multicolumn{2}{|c|}{ Dobutamine given in the first $24 \mathrm{~h}$} & $2(0.7)$ & $7(0.9)$ & 1.00 \\
\hline & \multicolumn{2}{|c|}{ Steroids given in the first $24 \mathrm{~h}$} & $71(23.4)$ & $142(18.1)$ & 0.048 \\
\hline \multirow[t]{2}{*}{ Admission } & \multicolumn{2}{|l|}{ Admitted to the GPU } & $201(66.1)$ & $503(64.2)$ & 0.561 \\
\hline & \multicolumn{2}{|l|}{ Admitted to the ICU } & $166(54.6)$ & $413(52.7)$ & 0.568 \\
\hline \multirow[t]{2}{*}{ Intubation } & \multicolumn{2}{|c|}{ Intubation in the first $24 \mathrm{~h}$} & $58(19.1)$ & $118(15.1)$ & 0.104 \\
\hline & \multicolumn{2}{|c|}{ Intubation in the first $48 \mathrm{~h}$} & 53 (17.5) & 70 (8.9) & $<0.0001$ \\
\hline \multicolumn{3}{|l|}{ Mortality in the ED } & $7(2.4)$ & $3(0.4)$ & 0.003 \\
\hline \multicolumn{3}{|l|}{ In hospital mortality } & $124(40.7)$ & $294(37.4)$ & 0.314 \\
\hline
\end{tabular}

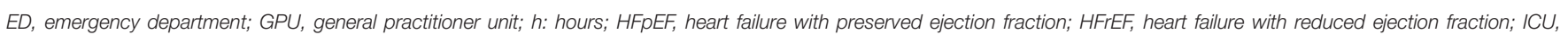
intensive care unit; $I$, intravenous; L, liters; LOS, length of stay; SD, standard deviation. 
TABLE 4 | Infection characteristics.

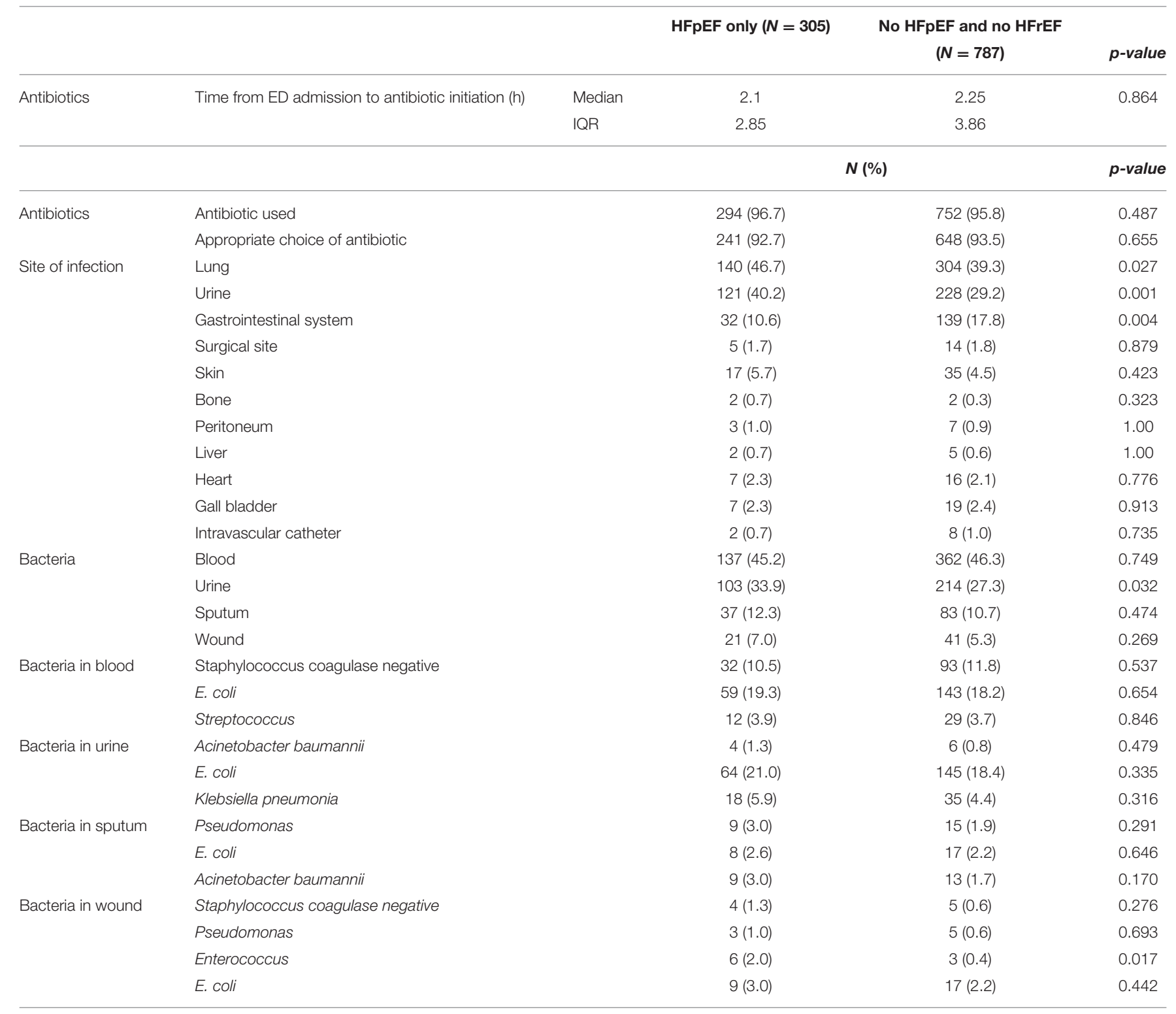

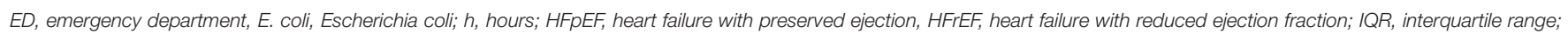
$S D$, standard deviation.

and ED mortality, and HFpEF and total LOS of in-hospital survivors, while taking into consideration all statistically and clinically relevant variables in the bivariate analysis (Tables 5-7).

There was no significant difference in in-hospital mortality between patients with $\mathrm{HFpEF}$ and patients without heart failure (OR 1.21, 95\% CI 0.90-1.62) after adjusting for confounders. Patients who underwent intubation in the first $24 \mathrm{~h}$ (OR 2.53, 95\% CI 1.74-3.69) and patients whose lungs were the site of infection (OR 1.78, 95\% CI 1.36-2.34) had higher odds of inhospital mortality. Moreover, for every unit increase of lactic acid at presentation (OR 1.10, 95\% CI 1.03-1.18), patients had higher odds of in-hospital mortality (Table 5). Patients with HFpEF had significantly higher odds of ED mortality compared to patients without heart failure (OR 9.07, 95\% CI 1.98-41.60) after adjusting for confounders (Table 6). After adjusting for comorbidities and other confounders, there was no significant difference in total LOS of in-hospital survivors with HFpEF compared to in-hospital survivors without heart failure $(\beta-42.20,95 \%$ CI -111.77-27.27) (Table 7).

\section{DISCUSSION}

Our results showed that HFpEF patients had a slightly increased in-hospital mortality as compared to the non-heart failure group; however, this difference was not statistically significant. On the other hand, septic HFpEF patients had a significantly 
TABLE 5 | Multivariate logistic regression of potential predictors of Hospital mortality.

\begin{tabular}{|c|c|c|}
\hline Variables & OR (95 \% Cl) & p-value \\
\hline \multicolumn{3}{|c|}{ Hospital mortality (reference: no) } \\
\hline HFpEF only & $1.21(0.90-1.62)$ & 0.21 \\
\hline Lactate at presentation & $1.10(1.03-1.18)$ & 0.004 \\
\hline Intubation first $24 \mathrm{~h}$ & 2.53 (1.74-3.69) & $<0.0001$ \\
\hline Site of infection-lung & $1.78(1.36-2.34)$ & $<0.0001$ \\
\hline \multicolumn{3}{|c|}{$\begin{array}{l}\text { Variables included in the model were. } \\
\text { Imposed: HFpEF - (reference: no HFpEF and no HFrEF). } \\
\text { Stepwise: age; gender (reference: female); vital signs heart rate (HR); smoking; chronic } \\
\text { kidney disease; hypertension; dyslipidemia; coronary artery disease; atrial fibrillation; } \\
\text { diabetes mellitus; chronic obstructive pulmonary disease (COPD); lactate at presentation; } \\
\text { procalcitonin; creatinine; phosphate; IV fluid first } 6 \mathrm{~h} \text {; } \mathrm{N} \text { fluid given in the first } 24 \mathrm{~h} \text {; } \\
\text { norepinephrine given in the first } 24 \mathrm{~h} \text {; steroids given in the first } 24 \mathrm{~h} \text {; intubation first } 24 \mathrm{~h} \text {; } \\
\text { intubation in the first } 48 \mathrm{~h} \text {; site of infection (lung; urine; gastrointestinal system), urine } \\
\text { bacteria; bacteria in wound-Enterococcus. }\end{array}$} \\
\hline
\end{tabular}

TABLE 6 | Multivariate logistic regression of potential predictors of Mortality in the ED.

\begin{tabular}{lcc}
\hline Variables & OR $(\mathbf{9 5} \% \mathbf{~ C l})$ & $\boldsymbol{p}$-value \\
\hline ED mortality (reference: no) & $9.07(1.98-41.60)$ & 0.005 \\
HFpEF only & $1.42(1.06-1.89)$ & 0.02 \\
Vital signs HR(increase by 10 units) & $23.45(4.61-119.25)$ & $<0.0001$ \\
Intubation first 24h & $0.11(0.02-0.66)$ & 0.02 \\
Site of infection-lung & $58.94(6.01-578.23)$ & $<0.0001$ \\
Bacteria in wound-Enterococcus & & \\
\hline
\end{tabular}

Variables included in the model were.

Imposed: HFpEF-(reference: no HFpEF and no HFrEF).

Stepwise: age; gender (reference: female); vital signs heart rate (HR); smoking; chronic kidney disease; hypertension; dyslipidemia; coronary artery disease; atrial fibrillation; diabetes mellitus; chronic obstructive pulmonary disease (COPD); lactate at presentation; procalcitonin; creatinine; phosphate; IV fluid first $6 h ; \mathrm{N}$ fluid given in the first $24 h$; norepinephrine given in the first $24 h$; steroids given in the first $24 h$; intubation first $24 h$; intubation in the first $48 \mathrm{~h}$; site of infection (lung; urine; gastrointestinal system), urine bacteria; bacteria in wound-Enterococcus.

$\mathrm{Cl}$, confidence interval; $\mathrm{ED}$, emergency department; $h$, hours; HFpEF, heart failure with preserved ejection fraction; HFrEF, heart failure with reduced ejection fraction; $N$, intravenous; OR, odds ratio.

higher ED mortality compared to septic patients without heart failure. Moreover, HFpEF patients were found to have 1.21 times the odds of hospital mortality compared to controls and 9 times the odds for ED mortality after adjusting for potential confounders. These results are in line with the literature, where a meta-analysis by Sanfilippo et al. found that septic patients with diastolic dysfunction had a significantly higher mortality rate than patients with no diastolic dysfunction (9). There are several proposed reasons for the increased mortality in HFpEF patients. First, the cytokines released in sepsis lead to worsening diastolic dysfunction and myocardial suppression, as well as profound vasoplegia and an increase in vascular permeability and capacitance $(8,9,11)$. This hypovolemic state is also accompanied by a reflex tachycardia, which worsens the LV filling, mainly by decreasing the diastolic time $(8,9,11)$. This cycle leads to a decreased cardiac output and poor perfusion which worsens
TABLE 7 | Multivariate linear regression of potential predictors of LOS of the survivors in hospital.

\begin{tabular}{lcc}
\hline Variables & $\boldsymbol{\beta}$ (95 \% Cl) & $\boldsymbol{p}$-value \\
\hline LOS & & \\
HFpEF only & $-42.20(-111.77 ; 27.37)$ & 0.23 \\
Vital signs HR(increase by 10 units) & $-14.57(-27.82 ;-1.31)$ & 0.03 \\
History of cancer & $89.62(23.90 ; 155.34)$ & 0.008 \\
IV fluid given in the first 24h & $21.37(7.12 ; 35.63)$ & 0.003 \\
\hline
\end{tabular}

Variables included in the model were.

Imposed: HFpEF-(reference: no HFpEF and no HFrEF).

Stepwise: age; gender (reference: female); vital signs heart rate (HR); smoking; chronic kidney disease; hypertension; dyslipidemia; end-stage renal disease; TIA or stroke; vascular disease; history of cancer; coronary artery disease; atrial fibrillation; diabetes mellitus; COPD; lactate at presentation; procalcitonin; creatinine; phosphate; IV fluid first $6 h$; IV fluid given in the first $24 h$; norepinephrine given in the first $24 \mathrm{~h}$; steroids given in the first $24 \mathrm{~h}$; intubation first $24 \mathrm{~h}$; intubation in the first $48 \mathrm{~h}$; site of infection (lung; urine; gastrointestinal system), urine bacteria; bacteria in wound-Enterococcus.

$\mathrm{Cl}$, confidence interval; $h$, hours; HFpEF, heart failure with preserved ejection fraction; HFrEF, heart failure with reduced ejection fraction; IV, intravenous.

organ failure. Landesberg et al. also conducted a retrospective study and found that diastolic dysfunction, in particular, when associated with a low cardiac output and stroke volume (SV) is a stronger predictor of early mortality in patients with sepsis and septic shock (6).

Second, sepsis also causes rhythm disturbances, and in a recent study, sepsis was an independent predictor of newonset atrial fibrillation (AF) (12). AF is common in HFpEF as it is identified in two-thirds of HFpEF patients, and its presence is associated with increased morbidity and mortality (13). This was reflected in our study, where HFpEF septic patients had a significantly higher percentage of atrial fibrillation as compared to non-heart failure patients. Aggressive fluid resuscitation remains one of the mainstays of sepsis management. The surviving sepsis campaign advocates for $30 \mathrm{cc} / \mathrm{kg}$ of fluid as part of the initial treatment of sepsis (14-16). However, aggressive fluid resuscitation has fallen out of favor and can be particularly hazardous in heart failure patients with preserved ejection fraction (17-20). Excessive fluid loading to the noncompliant LV may aggravate lung congestion and cardiogenic pulmonary edema which is common in sepsis (21-23). In our study, even though HFpEF patients received less fluids than controls (1.94 L vs. $2.41 \mathrm{~L} ; p<0.0001$ ), they had a higher rate of intubation, probably due to cardiogenic pulmonary edema. Due to the impaired LV relaxation and the sepsis-induced myocardial suppression, these patients are very sensitive to fluid and can develop pulmonary edema rapidly.

It is interesting to note that there was no difference in hospital lengths of stay between both groups nor were there any differences in the lactate acid level, the site of infections, or the most commonly isolated bacteria. One possible explanation for the length of stay similarity could be that HFpEF has a higher mortality early on in the course of sepsis, as witnessed by their high ED mortality in our cohort and their increased intubation rates. However, if they overcome this early period, HFpEF patients tend to have a similar hospital course like non-heart failure patients. Nonetheless, emergency physicians should be 
aware of their increased mortality. The authors recommend early aggressive measures and early coordination and consultation with cardiology specialists as well as critical care specialists when dealing with a septic HFpEF patient.

\section{Limitations}

Given that this was a retrospective chart review cohort study, the most important limitation is the biases associated with this kind of study. In order to minimize those, frequent meetings were held between the principal investigator and research assistants to standardize the way in which data were collected, entered, and cleaned. This study is from a referral academic center ED that deals with regional complicated cases, which could explain the increased mortality seen in both cohorts and could limit the generalizability of the results. Second, the delay in antibiotic administration (median: 2.1 vs. $2.25 \mathrm{~h}$; interquartile range and interquartile range (IQR): 2.85 vs. $3.86 \mathrm{~h}$ ) might have led to the increased morality, as it has been shown in several studies $(20,24,25)$. It is important to note that both cohorts were found to be unmatched in terms of comorbidities, making our results difficult to interpret. In an effort to correct for this, all statistically and clinically significant characteristics on bivariate analysis were controlled for in the multivariable analysis in order to minimize confounding variables. It is important to note that our secondary outcomes, with the exception of ED mortality and LOS among in-hospital survivors, are the result of univariate analysis and as such are subject to confounders. As such, our results should be interpreted with caution. Moreover, patients spent an extended period of time in the ED overall $(15.77 \pm 18.86 \mathrm{~h}$ for HFpEF patients and $16.66 \pm 21.93$ for patients without heart failure); this should be kept in mind as it can limit the generalizability of the results. Another limitation of the study is the exclusion of patients with HFrEF. We understand that this is a substantial subgroup of patients with heart failure, but we chose to exclude these patients because the aim was to look at septic HFpEF and study their outcome as the literature on this topic is scarce. Furthermore, we conducted a study looking at the mortality of HFrEF septic patients as compared to controls at our institution and we were able to show that HFrEF septic patients had 2.5 times the odds for in-hospital mortality than patients without heart failure (26). Finally, while our study looked at HFpEF patients, it is important to note that our control patients might have had sepsis-induced

\section{REFERENCES}

1. Roger VL. Epidemiology of heart failure. Circ Res. (2013) 113:64659. doi: 10.1161/CIRCRESAHA.113.300268

2. Borlaug BA, Paulus WJ. Heart failure with preserved ejection fraction: pathophysiology, diagnosis, and treatment. Eur Heart J. (2011) 32:6709. doi: 10.1093/eurheartj/ehq426

3. Dunlay SM, Roger VL. Understanding the epidemic of heart failure: past, present, and future. Curr Heart Fail Rep. (2014) 11:404-15. doi: 10.1007/s11897-014-0220-x

4. Sharma K, Kass DA. Heart failure with preserved ejection fraction: mechanisms, clinical features, and therapies. Circ Res. (2014) 115:79-96. doi: 10.1161/CIRCRESAHA.115. diastolic dysfunction without heart failure symptoms and this might have increased their mortality (27).

\section{CONCLUSION}

Septic HFpEF patients experienced an increased rate of ED mortality, intubation, and steroid use compared to controls. This indicates increased vulnerability for this subcategory of patients. It is important to note that, because of the increased risk of lung congestion and cardiopulmonary edema in these patients, it is preferable to start vasopressors early on for hemodynamic support than to potentially overwhelm them with fluids. Septic HFpEF patients should benefit from a multidisciplinary approach in order to improve their outcome. This is of particular importance in the early phase of the disease given the increased risk of ED noted in our study. ED physicians should promptly identify these patients and aim for individualized and optimal care.

\section{DATA AVAILABILITY STATEMENT}

The datasets generated for this study are available on request to the corresponding author.

\section{ETHICS STATEMENT}

The studies involving human participants were reviewed and approved by Institutional Review Board of the American University of Beirut. Written informed consent for participation was not required for this study in accordance with the national legislation and the institutional requirements.

\section{AUTHOR CONTRIBUTIONS}

RC and GD made substantial contributions to the conception and design of the study. RC, GD, IB, RS, AS, MS, MM, and HT were responsible for the acquisition of data, analysis, and interpretation of data. RC, GD, IB, SJ, and RS were involved in drafting the manuscript. GD, RC, IB, and RS were responsible for revising the manuscript critically for important intellectual content. RC and GD took responsibility for the paper as a whole. All authors contributed substantially to its revision and approved the submitted version.
5. Hajj J, Blaine N, Salavaci J, Jacoby D. The "centrality of sepsis": a review on incidence, mortality, and cost of care. Healthcare. (2018) 6:90. doi: 10.3390/healthcare6030090

6. Landesberg G, Gilon D, Meroz Y, Georgieva M, Levin PD, Goodman S, et al. Diastolic dysfunction and mortality in severe sepsis and septic shock. Eur Heart J. (2012) 33:895-903. doi: 10.1093/eurheartj/ehr351

7. Luhr R, Cao Y, Soderquist B, Cajander S. Trends in sepsis mortality over time in randomised sepsis trials: a systematic literature review and metaanalysis of mortality in the control arm, 2002-2016. Crit Care. (2019) 23:241. doi: 10.1186/s13054-019-2528-0

8. Drosatos K, Lymperopoulos A, Kennel PJ, Pollak N, Schulze PC, Goldberg IJ. Pathophysiology of sepsis-related cardiac dysfunction: driven by inflammation, energy mismanagement, or both? Curr Heart Fail Rep. (2015) 12:130-40. doi: 10.1007/s11897-014-0247-z 
9. Sanfilippo F, Corredor C, Fletcher N, Landesberg G, Benedetto U, Foex P, et al. Diastolic dysfunction and mortality in septic patients: a systematic review and meta-analysis. Intensive Care Med. (2015) 41:100413. doi: 10.1007/s00134-015-3748-7

10. Singer M, Deutschman CS, Seymour CW, Shankar-Hari M, Annane D, Bauer $\mathrm{M}$, et al. The third international consensus definitions for sepsis and septic shock (Sepsis-3). JAMA. (2016) 315:801-10. doi: 10.1001/jama.2016.0287

11. Nagueh SF, Smiseth OA, Appleton CP, Byrd BF, 3rd, Dokainish H, Edvardsen $\mathrm{T}$, et al. Recommendations for the evaluation of left ventricular diastolic function by echocardiography: an update from the American society of echocardiography and the European association of cardiovascular imaging. J Am Soc Echocardiogr. (2016) 29:277-314. doi: 10.1016/j.echo.2016.01.011

12. Makrygiannis SS, Margariti A, Rizikou D, Lampakis M, Vangelis S, Ampartzidou OS, et al. Incidence and predictors of new-onset atrial fibrillation in noncardiac intensive care unit patients. J Crit Care. (2014) 29:697.e1-5. doi: 10.1016/j.jcrc.2014.03.029

13. Zakeri R, Chamberlain AM, Roger VL, Redfield MM. Temporal relationship and prognostic significance of atrial fibrillation in heart failure patients with preserved ejection fraction: a community-based study. Circulation. (2013) 128:1085-93. doi: 10.1161/CIRCULATIONAHA.113. 001475

14. Levy MM, Evans LE, Rhodes A. The surviving sepsis campaign bundle. Crit Care Med. (2018) 46:997-1000. doi: 10.1097/CCM.0000000000003119

15. Rhodes A, Evans LE, Alhazzani W, Levy MM, Antonelli M, Ferrer R, et al. Surviving sepsis campaign: international guidelines for management of sepsis and septic shock: 2016. Intensive Care Med. (2017) 43:304-77. doi: 10.1097/CCM.0000000000002255

16. Rivers E, Nguyen B, Havstad S, Ressler J, Muzzin A, Knoblich B, et al. Early goal-directed therapy in the treatment of severe sepsis and septic shock. $N$ Engl J Med. (2001) 345:1368-77. doi: 10.1056/NEJMoa010307

17. ProCESS Investigators, Yealy DM, Kellum JA, Barnato AE, Weissfeld LA, Pike F, et al. A randomized trial of protocol-based care for early septic shock. N Engl J Med. (2014) 370:1683-93. doi: 10.1056/NEJMoa 1401602

18. Investigators A, Group ACT, Peake SL, Delaney A, Bailey M, Bellomo $\mathrm{R}$, et al. Goal-directed resuscitation for patients with early septic shock. N Engl J Med. (2014) 371:1496-506. doi: 10.1056/NEJMoa14 04380

19. Mouncey PR, Osborn TM, Power GS, Harrison DA, Sadique MZ, Grieve RD, et al. Trial of early, goal-directed resuscitation for septic shock. $N$ Engl J Med. (2015) 372:1301-11. doi: 10.1056/NEJMoa1500896
20. Osborn TM. Severe sepsis and septic shock trials (ProCESS, ARISE, ProMISe): what is optimal resuscitation? Crit Care Clin. (2017) 33:32344. doi: 10.1016/j.ccc.2016.12.004

21. Lee WL, Slutsky AS. Sepsis and endothelial permeability. N Engl J Med. (2010) 363:689-91. doi: 10.1056/NEJMcibr1007320

22. Schneider AJ, Teule GJ, Groeneveld AB, Nauta J, Heidendal GA, Thijs LG. Biventricular performance during volume loading in patients with early septic shock, with emphasis on the right ventricle: a combined hemodynamic and radionuclide study. Am Heart J. (1988) 116(1 Pt 1):10312. doi: 10.1016/0002-8703(88)90256-6

23. Westermann D, Kasner M, Steendijk P, Spillmann F, Riad A, Weitmann $\mathrm{K}$, et al. Role of left ventricular stiffness in heart failure with normal ejection fraction. Circulation. (2008) 117:2051-60. doi: 10.1161/CIRCULATIONAHA.107.716886

24. Kumar A, Roberts D, Wood KE, Light B, Parrillo JE, Sharma S, et al. Duration of hypotension before initiation of effective antimicrobial therapy is the critical determinant of survival in human septic shock. Crit Care Med. (2006) 34:1589-96. doi: 10.1097/01.CCM.0000217961.75225.E9

25. Sterling SA, Miller WR, Pryor J, Puskarich MA, Jones AE. The impact of timing of antibiotics on outcomes in severe sepsis and septic shock: a systematic review and meta-analysis. Crit Care Med. (2015) 43:190715. doi: 10.1097/CCM.0000000000001142

26. Abou Dagher G, Hajjar K, Khoury C, El Hajj N, Kanso M, Makki $\mathrm{M}$, et al. Outcomes of patients with systolic heart failure presenting with sepsis to the emergency department of a tertiary hospital: a retrospective chart review study from Lebanon. BMJ Open. (2018) 8:e022185. doi: 10.1136/bmjopen-2018-022185

27. Poelaert J. Diastolic dysfunction and sepsis: the devil is in the detail. $\mathrm{Br} J$ Anaesth. (2017) 119:555-7. doi: 10.1093/bja/aex325

Conflict of Interest: The authors declare that the research was conducted in the absence of any commercial or financial relationships that could be construed as a potential conflict of interest.

Copyright (C) 2020 Chebl, Berbari, Safa, Shami, Sabra, Jamali, Makki, Tamim and Dagher. This is an open-access article distributed under the terms of the Creative Commons Attribution License (CC BY). The use, distribution or reproduction in other forums is permitted, provided the original author(s) and the copyright owner(s) are credited and that the original publication in this journal is cited, in accordance with accepted academic practice. No use, distribution or reproduction is permitted which does not comply with these terms. 